INPLASY

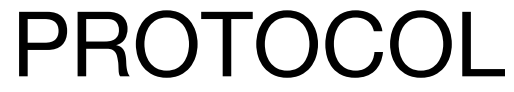

To cite: Cui et al. A metaanalysis of the association between trisomy 12 and prognosis of chronic lymphocytic leukemia. Inplasy protocol 202060031. doi: 10.37766/inplasy2020.6.0031

Received: 09 June 2020

Published: 10 June 2020

Corresponding author: Chuanzhong Mei

Xmeichzh@sina.com

Author Affiliation:

ChuanZhong Mei:+86

18725528276

Support: National natural science found

Review Stage at time of this submission: Formal screening of search results against eligibility criteria.

Conflicts of interest: None.

\section{A meta-analysis of the association between trisomy 12 and prognosis of chronic lymphocytic leukemia}

Cui, D'; Zheng, W2; Zhang, W3; Zhou, J4; Mei, C. ${ }^{5}$

Review question / Objective: Trisomy 12 (+12) was one of the most common chromosomal abnormality reported in CLL patients, which occured in $16-25 \%$ of cases. Although the discovery of +12 has been extensively documented and many studies on the relationship between +12 and the prognosis of chronic lymphocytic leukemia(CLL), the results were still controversial. Thus, we performed a meta-analysis to clarify the prognostic value of trisomy 12 in CLL.

Condition being studied: With the emergence of the fluorescence in situ hybridization and the next-generation sequencing technology, cytogenetic markers have begun to play an important role, especially chromosome aberrations, which affect the clinical prognostic on patients with CLL through various ways. Trisomy $12(+12)$ is one of the most common chromosomal abnormality reported in CLL patients, which occurs in $16-25 \%$ of cases. Many studies have confirmed the clinical and biological characteristics of +12 , however, the prognostic relevance of +12 in CLL remains a matter of debate. Therefore, we explored the relationship between trisomy 12 and CLL, as well as its influence on prognosis through meta-analysis.

INPLASY registration number: This protocol was registered with the International Platform of Registered Systematic Review and Meta-Analysis Protocols (INPLASY) on 10 June 2020 and was last updated on 10 June 2020 (registration number INPLASY202060031).

\section{INTRODUCTION}

Review question / Objective: Trisomy 12 (+12) was one of the most common chromosomal abnormality reported in CLL patients, which occured in $16-25 \%$ of cases. Although the discovery of +12 has been extensively documented and many studies on the relationship between +12 and the prognosis of chronic lymphocytic leukemia(CLL), the results were still controversial. Thus, we performed a meta- 
analysis to clarify the prognostic value of trisomy 12 in CLL.

Condition being studied: With the emergence of the fluorescence in situ hybridization and the next-generation sequencing technology, cytogenetic markers have begun to play an important role, especially chromosome aberrations, which affect the clinical prognostic on patients with CLL through various ways. Trisomy $12(+12)$ is one of the most common chromosomal abnormality reported in CLL patients, which occurs in $16-25 \%$ of cases. Many studies have confirmed the clinical and biological characteristics of +12 , however, the prognostic relevance of +12 in CLL remains a matter of debate. Therefore, we explored the relationship between trisomy 12 and CLL, as well as its influence on prognosis through meta-analysis.

\section{METHODS}

Participant or population: The patients of chronic lymphoblastic leukemia.

Intervention: The CLL patients with trisomy 12.

Comparator: The CLL patients without trisomy.

Study designs to be included: Prospective, retrospective, or randomized clinical trial studies.

Eligibility criteria: . Prospective, retrospective, or clinical trial studies published on or before May 1, 2020. II The research object was associated with CLL and trisomy 12. III. Provided prognosis information for CLL patients with trisomy 12, outcome indicators at least one of included hazard ratios (HRs) of overall survival (OS), progression-free survival (PFS), and time to first treatment (TTFT) with their $95 \%$ confidence intervals $195 \%$ Cls).

Information sources: Three databases, PubMed, Web of Science and Science direct, were retrieved in this paper, and the retrieval time was up to May 1, 2020.
Main outcome(s): The hazard ratios (HRs) of overall survival (OS), progression-free survival (PFS), and time to first treatment (TTFT) with their 95\% confidence intervals (95\% Cls).

Quality assessment / Risk of bias analysis: Publication bias was quantitatively analyzed by Begg's test and Egger's linear regression, and the reliability of metaanalysis results were tested by sensitivity analysis excluding individual studies. A pvalue of $<0.05$ was considered significant publication bias.

Strategy of data synthesis: This metaanalysis was performed with Stata 12.0, HRs and $95 \% \mathrm{Cls}$ included of various studies were combined to draw forest maps and evaluate the heterogeneity of results. If $\mathrm{P}>0.01$ or $\mathbf{1} 2<50 \%$, there was no significant heterogeneity among the studies, and the fixed effect model was used for statistical analysis. If $P<0.01$ and I $2 \geq 50 \%$, indicating significant heterogeneity between studies, we used the random effects model to analysis.

Subgroup analysis: Subgroup analysis is performed according to region, sample size, median follow-up time and with or without Notch1 mutation.

Sensibility analysis: The sensitivity analysis of the effect of trisomy 12 on the OS , PFS and TTFT was carried out by sequentially excluding studies.

\section{Language: English.}

Country(ies) involved: UK; USA; Italy; Czech; French; Austria; Denmark; Taiwan; Canada; Netherlands.

Keywords: chronic lymphocytic leukemia; Trisomy 12; Notch1 mutataion; prognosis.

Contributions of each author:

Author 1 - Di Cui - Author1 finished the conception and design. Than author1 independently screened the titles and abstracts of articles identified by the literature search, retrieved potentially relevant studies and determined study 
eligibility. Last, Author1 drafted the manuscript.

Author 2 - Weiwei Zheng - Author 2 has the same contribution as author 1 .

Author 3 - Wenjing Zhang - Author3 finish the acquisition of data, analysis and interpretation of data.

Author 4 - Jie Zhou - Author4 make the charts and tables.

Author 5 - Chuanzhong Mei - Author 5 complete the review and correction of the paper. 